\title{
Magnetic-field-induced crossover from non-Fermi to Fermi liquid at the quantum critical point of $\mathrm{YbCu}_{5-x} \mathrm{Au}_{x}$
}

\author{
P. Carretta, ${ }^{1}$ R. Pasero, ${ }^{2}$ M. Giovannini, ${ }^{2}$ and C. Baines ${ }^{3}$ \\ ${ }^{1}$ Dipartimento di Fisica "A. Volta," University of Pavia, Via Bassi 6, I-27100 Pavia, Italy \\ ${ }^{2}$ Dipartimento di Chimica e Chimica Industriale, University of Genova, I-16146 Genova, Italy \\ and LAMIA-INFM-CNR, Corso Perrone 24, I-16152 Genova, Italy \\ ${ }^{3}$ Laboratory for Muon Spin Spectroscopy, S $\mu$ S Swiss Muon Source, PSI, CH-5232 Villigen, Switzerland \\ (Received 15 September 2008; revised manuscript received 5 November 2008; published 6 January 2009)

\begin{abstract}
The temperature $(T)$ dependence of the muon and ${ }^{63} \mathrm{Cu}$ nuclear spin-lattice relaxation rates $1 / T_{1}$ in $\mathrm{YbCu}_{4.4} \mathrm{Au}_{0.6}$ is reported over nearly four decades. It is shown that for $T \rightarrow 01 / T_{1}$ diverges following the behavior predicted by the self-consistent renormalization (SCR) theory for a ferromagnetic quantum critical point. On the other hand, the static uniform susceptibility $\chi_{s}$ is observed to diverge as $T^{-2 / 3}$ and $1 / T_{1} T \propto \chi_{s}^{2}$, a behavior which is not accounted for by SCR theory. The application of a magnetic field $H$ is observed to induce a crossover to a Fermi-liquid behavior and for $T \rightarrow 01 / T_{1}$ is found to obey the scaling law $1 / T_{1}(H)$ $=1 / T_{1}(0)\left[1+\left(\mu_{B} H / k_{B} T\right)^{2}\right]^{-1}$.
\end{abstract}

DOI: 10.1103/PhysRevB.79.020401

PACS number(s): 76.60.Es, 71.27.+a, 75.40.Gb

Strongly correlated electron systems with competing interactions are known to show rather rich phase diagrams, with crossovers or phase transitions which depend on the relative magnitude of the competing energy scales. A paradigmatic example is represented by heavy-fermion intermetallic compounds, where a quantum phase transition between Fermi liquid (FL) and magnetic ground states is typically observed upon varying the single-ion Kondo coupling $J$ and the density of states at the Fermi level $D\left(E_{F}\right) .{ }^{1}$ The modification of these two parameters affects both the coherence temperature $T^{*}$, below which the $f$ electrons delocalize and a FL behavior is observed, and the transition temperature to a magnetic long-range order, which is determined by Ruderman-Kittel-Kasuya-Yosida (RKKY) interaction. ${ }^{2}$ At the quantum critical point (QCP) $T^{*} \rightarrow 0$, the Fermi-liquid regime is never attained and a rather peculiar behavior of the response functions is observed down to $T \rightarrow 0$, the so-called non-Fermi-liquid (NFL) regime. The QCP can be tuned by different parameters, such as the chemical composition, the pressure, and the magnetic field, which control the hybridization between $f$ and $s$ electron orbitals, i.e., $J$ and $D\left(E_{F}\right){ }^{3}$ In spite of the significant experimental efforts, an overall understanding of how the dynamical spin susceptibility behaves in the NFL regime on approaching the QCP and how it is affected by external parameters, such as the magnetic field, is still missing.

$\mathrm{YbCu}_{5-x} \mathrm{Au}_{x}$ is a heavy-fermion intermetallic compound which has been studied in recent years mostly with techniques of macroscopic character, ranging from specific heat to magnetization and resistivity measurements. ${ }^{4-6}$ On the basis of these experimental results a tentative phase diagram as a function of $x$ has been outlined. The coherence temperature $T^{*}$, which for $x=0$ was estimated to be around $5 \mathrm{~K}$, vanishes around $x \simeq 0.4$, where a quantum phase transition to a longrange magnetic order is expected. ${ }^{4}$ Still, it has to be pointed out that the transition temperature to the magnetically ordered phase for $x>0.5$ has been determined just from the change in slope in the resistivity vs temperature, ${ }^{4}$ raising some doubts on the accuracy of its estimate. Moreover, a careful analysis of the chemical and structural properties of $\mathrm{YbCu}_{5-x} \mathrm{Au}_{x}$ solid solutions ${ }^{7}$ has shown that homogeneous compounds with cubic $\mathrm{AuBe}_{5}$-type structure can be grown at ambient pressure only for $x \geq 0.4$, questioning some of the previous findings.

Here we will show, on the basis of zero-field and longitudinal field muon spin relaxations $(\mu \mathrm{SR})$, nuclear quadrupole resonance (NQR), and magnetization measurements, that for $x \simeq 0.6$ a ferromagnetic QCP is attained. Moreover, it will be shown that the $T$ dependence of the muon spin-lattice relaxation rate $1 / T_{1}^{\mu}$ for $T \rightarrow 0$ can be suitably described within the self-consistent renormalization (SCR) theory developed by Moriya. ${ }^{8}$ At temperatures above $1 \mathrm{~K}$ both $1 / T_{1}^{\mu}$ and the copper nuclear spin-lattice relaxation rate $1 / T_{1}^{63}$ are observed to scale with the square of the static uniform susceptibility. Hereafter, with $1 / T_{1}$ we shall refer both to the muon and nuclear spin-lattice relaxation rates, unless specified. Finally, it was found that the application of a magnetic field $H$ leads to a crossover from the critical NFL to a FL behavior and to a significant reduction in the relaxation rate.

The experiments were performed on $\mathrm{YbCu}_{5-x} \mathrm{Au}_{x}$ powders grown according to the procedure reported in Ref. 7. $\mu \mathrm{SR}$ experiments were performed at PSI Swiss muon source on LTF beamline. In order to reduce the background contamination, when the decay rate of the muon polarization $1 / T_{1}^{\mu} \leq 1 \mu \mathrm{s}^{-1}$ the data acquisition was performed in MORE mode. ${ }^{9}$ The decay of the muon polarization could be nicely fitted by

$$
P_{\mu}(t)=A \exp \left[-\left(t / T_{1}^{\mu}\right)^{\beta}\right]+B,
$$

with an initial asymmetry $(A+B) \simeq 24 \%$ over all the explored $T$ range (Fig. 1), while the exponent $\beta$ was found to slowly decrease from $\beta \simeq 0.5$ for $T=7 \mathrm{~K}$ to $\beta \simeq 0.3$ for $T=20 \mathrm{mK}$. Here $B$, a few percent, is the background contribution due to the sample holder and cryostat environment. No evidence of a transition to a magnetically ordered phase was observed down to $20 \mathrm{mK}$, at variance with previous findings based on resistivity measurements. ${ }^{4}$ As we shall see 


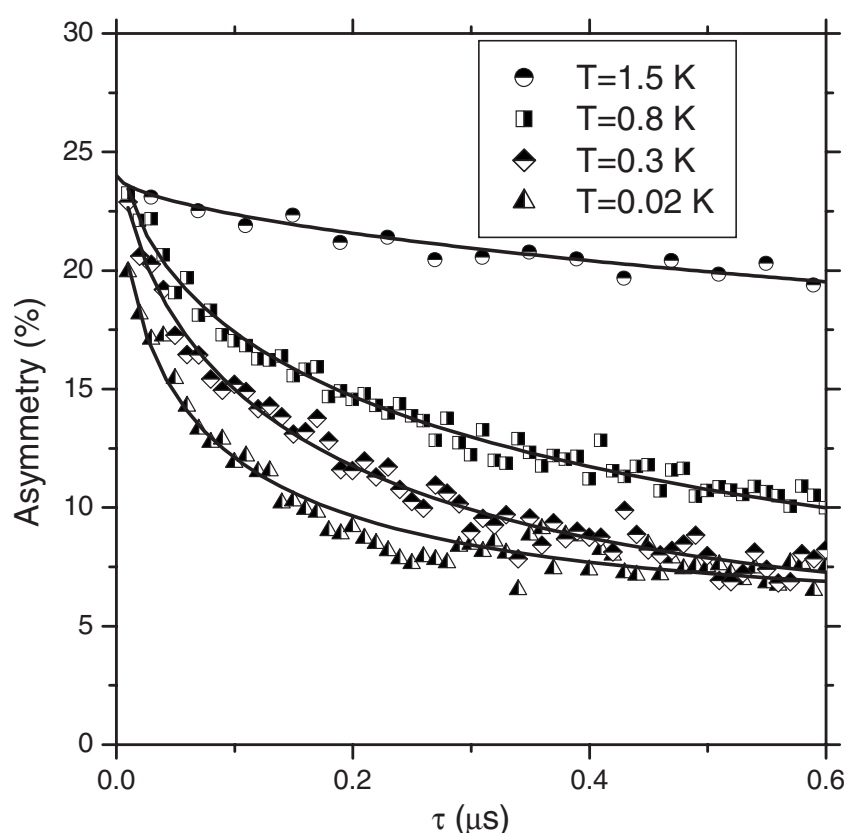

FIG. 1. Decay of the muon polarization in $\mathrm{YbCu}_{4.4} \mathrm{Au}_{0.6}$ in zero field at four different temperatures. The solid lines are the best fit according to Eq. (1).

from the magnetic field dependence of $1 / T_{1}^{\mu}$, the muon relaxation is dynamical, namely, driven by spin fluctuations and not by a static field distribution.

${ }^{63} \mathrm{Cu}$ NQR $1 / T_{1}^{63}$ measurements were performed using a standard saturation recovery pulse sequence. The recovery law of the nuclear magnetization after the saturating sequence could be fitted by a single exponential $y(t)=\exp \left(-3 t / T_{1}^{63}\right)$ over more than a decade. The ${ }^{63,65} \mathrm{Cu}$ NQR spectrum, centered around $10 \mathrm{MHz}$, is broader (full width at half maximum $\simeq 2 \mathrm{MHz}$ ) than the one reported for $x=0,{ }^{10}$ but no significant variation in ${ }^{63} \mathrm{Cu} 1 / T_{1}$ was detected through all the spectrum. Finally, the magnetization measurements were performed in a magnetic field $H=100 \mathrm{G}$ and the static uniform susceptibility was derived from the ratio $\chi_{s}=M / H$.

In Fig. 2 the $T$ dependence of $1 / T_{1} T$ is reported over nearly four decades. First of all one notices a trend quite different from the one reported for $x=0,{ }^{10}$ where a FL ground state is present and $1 / T_{1} T$ becomes constant for $T \rightarrow 0$. Moreover, in Fig. 2 two other relevant aspects are evidenced: $1 / T_{1} T$ scales with $\chi_{s}^{2}$ and, for $T \rightarrow 0,1 / T_{1} T \propto T^{-4 / 3}$. Accordingly, one has to expect that at low $T \chi_{s} \propto T^{-2 / 3}$. Indeed, from Fig. 3 one notices that in the low- $T$ range the static uniform susceptibility $\chi_{s}=C /\left(T^{2 / 3}+\Theta^{2 / 3}\right)$ for $x=0.6$, while this scaling is not so well obeyed for lower or higher Au content. Moreover, one can notice that for $x=0.6$ the Curie-Weiss temperature $\Theta$ vanishes. This indicates that if $x=0.6$ corresponds to the critical doping the quantum phase transition should separate a FL from a ferromagnetic ground state. The observation of a Curie-Weiss law, characterized by an anomalous exponent $\alpha$ (here $\alpha=2 / 3$ ), is common to several heavy-fermion compounds close to a QCP. ${ }^{12}$ In particular, in $\mathrm{YbRh}_{2}\left(\mathrm{Si}_{0.95} \mathrm{Ge}_{0.05}\right)_{2}$ where a ferromagnetic QCP is present ${ }^{13}$ $\chi_{s} \propto T^{-0.6}$, a behavior very similar to the one found for

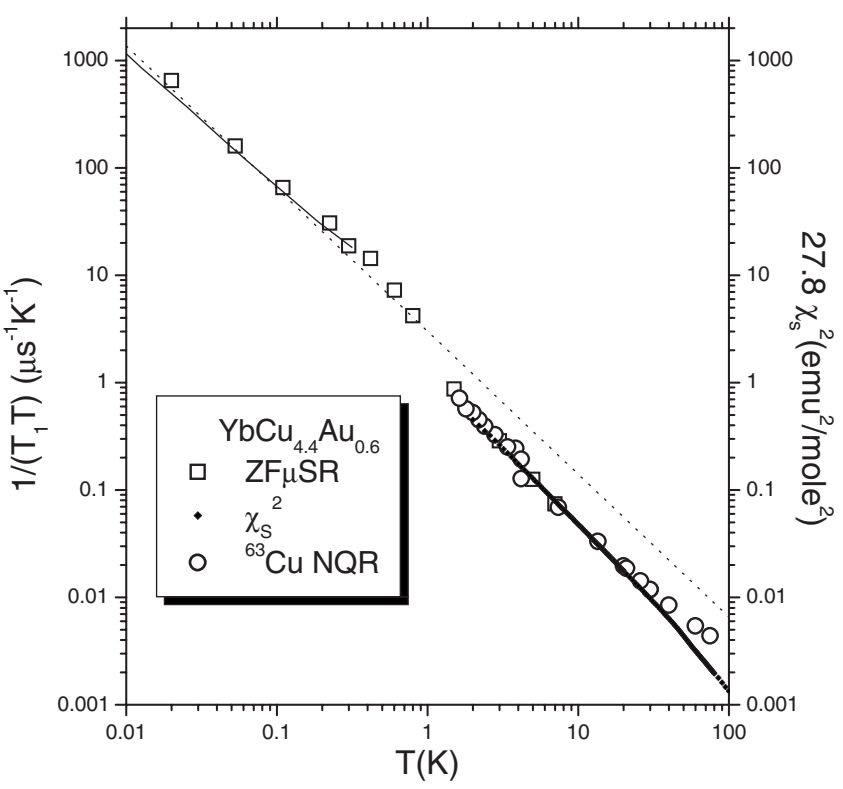

FIG. 2. $T$ dependence of the muon and nuclear spin-lattice relaxation rates divided by temperature in $\mathrm{YbCu}_{4.4} \mathrm{Au}_{0.6}$ for $H=0$. The nuclear spin-lattice relaxation rate has been rescaled by a factor of 236 in order to match muon $1 / T_{1} T$ in the $T$ range where both sets of data are present. The solid line shows the low- $T$ numerical results by Ishigaki and Moriya (Ref. 11). The dotted line shows the power law $T^{-4 / 3}$.

$\mathrm{YbCu}_{4.4} \mathrm{Au}_{0.6}$. It is worth mentioning that in $\mathrm{YbRh}_{2}\left(\mathrm{Si}_{0.95} \mathrm{Ge}_{0.05}\right)_{2}$ a ferromagnetic QCP appears in spite of the proximity to an antiferromagnetic ground state, ${ }^{13}$ a situation analogous to the one observed for $\mathrm{YbCu}_{5-x} \mathrm{Au}_{x}$, which for $x=1$ is known to order antiferromagnetically. ${ }^{14}$ Hence, a modification of the critical wave vector has to be expected upon approaching the critical doping $x=0.6$.

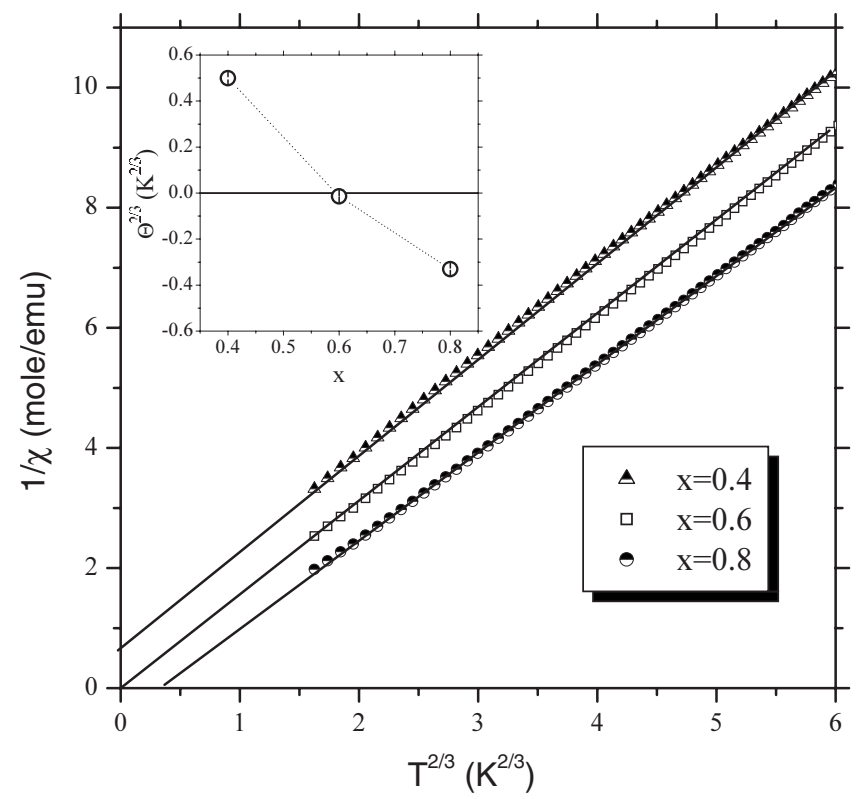

FIG. 3. Inverse of the molar spin susceptibility in $\mathrm{YbCu}_{5-x} \mathrm{Au}_{x}$ vs $T^{2 / 3}$. One notices that for $x=0.6$ a good scaling is found and that the Curie-Weiss temperature vanishes. In the inset $\Theta^{2 / 3}$, as estimated from the fit of $\chi_{s}=C /\left(T^{2 / 3}+\Theta^{2 / 3}\right)$, is reported. 
Let us now discuss the low-energy excitations in light of the results obtained from $1 / T_{1}$ measurements. In the presence of a relaxation mechanism driven by spin fluctuations one can write $1 / T_{1}$ in terms of the imaginary part of the dynamical spin susceptibility $\chi^{\prime \prime}\left(\vec{q}, \omega_{R} \rightarrow 0\right)$,

$$
\frac{1}{T_{1}}=\frac{\gamma^{2} k_{B} T}{2 N} \sum_{\vec{q}}\left|A_{\vec{q}}\right|^{2} \frac{\chi^{\prime \prime}\left(\vec{q}, \omega_{R}\right)}{\omega_{R}},
$$

where $\gamma$ is the gyromagnetic ratio and $\left|A_{\vec{q}}\right|^{2}$ is the form factor, giving the hyperfine coupling of the muon (or nuclei) with the spin excitations at wave vector $\vec{q}$. From Fig. 2 one notices that ${ }^{63} \mathrm{Cu}$ and $\mu^{+} 1 / T_{1}$ differ by a factor of 236 . From the above equations, taking into account that $\left(\gamma_{\mu} / \gamma_{63}\right)^{2}$ $\simeq 144$, one realizes that the hyperfine couplings of ${ }^{63} \mathrm{Cu} \mathrm{nu}-$ clei and of the $\mu^{+}$are quite similar.

Following Ishigaki and Moriya ${ }^{15}$ it is convenient to write the dynamical spin susceptibility in terms of two parameters $T_{0}$ and $T_{A}$ which characterize the width of the spin excitation spectra in frequency and $\vec{q}$, respectively. For ferromagnetic correlations one has ${ }^{11,15}$

$$
\chi(q, \omega)=\frac{\pi T_{0}}{\alpha_{Q} T_{A}} \frac{x}{k_{B} 2 \pi T_{0} x\left(y+x^{2}\right)-i \omega \hbar}
$$

where $x=q / q_{D}$, with $q_{D}$ as a Debye-type cut-off wave vector, $\alpha_{Q}$ as a dimensionless interaction constant close to unity for a strongly correlated system, and $y=N_{A} / 2 \alpha_{Q} k_{B} T_{A} \chi_{s}$. Here the susceptibility is per spin and in $4 \mu_{B}^{2}$ units and has the dimensions of the inverse of energy, while $T_{A}$ and $T_{0}$ are in kelvin. From the previous expression one can derive $\chi^{\prime \prime}\left(\vec{q}, \omega_{R}\right) / \omega_{R}$ by taking the limit $\omega_{R} \rightarrow 0$ since $\hbar \omega_{R} \ll k_{B} T$. Then, by integrating $\chi^{\prime \prime}\left(\vec{q}, \omega_{R}\right) / \omega_{R}$ in $\vec{q}$, over a sphere of radius $q_{D}$, one derives

$$
\frac{1}{T_{1}}=\frac{\gamma^{2} A^{2}}{2} T \frac{3 \hbar}{4 \pi k_{B} T_{A} T_{0}} \frac{1}{\alpha_{Q}} \frac{1}{2 y(1+y)} .
$$

Now, if $T_{A} \gg T$ in the $T$ range of interest then $y \ll 1$ (Refs. 11 and 15) and one can simplify the previous expression in the form

$$
\frac{1}{T_{1}} \simeq \gamma^{2} A^{2} \frac{3 \hbar}{8 \pi}\left(\frac{T}{T_{0}}\right) \frac{\chi_{s}}{N_{A}}
$$

This expression corresponds to the one derived by Ishigaki and Moriya, ${ }^{11,15}$ provided that one takes into account that their hyperfine coupling constants are in $\mathrm{kOe} / \mu_{B}$. This is the behavior typically observed in the presence of a magnetic ground state. ${ }^{16}$ For a three-dimensional system approaching a ferromagnetic QCP $\chi_{s}$ is expected to diverge as $T^{-4 / 3}$ at low temperature and, accordingly, $1 / T_{1} \propto T^{-1 / 3}$, exactly the behavior observed in our measurements. However, it has to be pointed out that SCR theory would predict a spin-lattice relaxation rate $1 / T_{1} \propto T \chi_{s}$, at variance with the experimental findings. Therefore, although the $T$ dependence of $1 / T_{1}$ seems to agree with predictions of SCR theory we do not find a full consistency of our experimental findings with the theory. As we shall see in the next paragraph, the $H$ dependence of $1 / T_{1}$ can hardly also be explained by SCR theory.

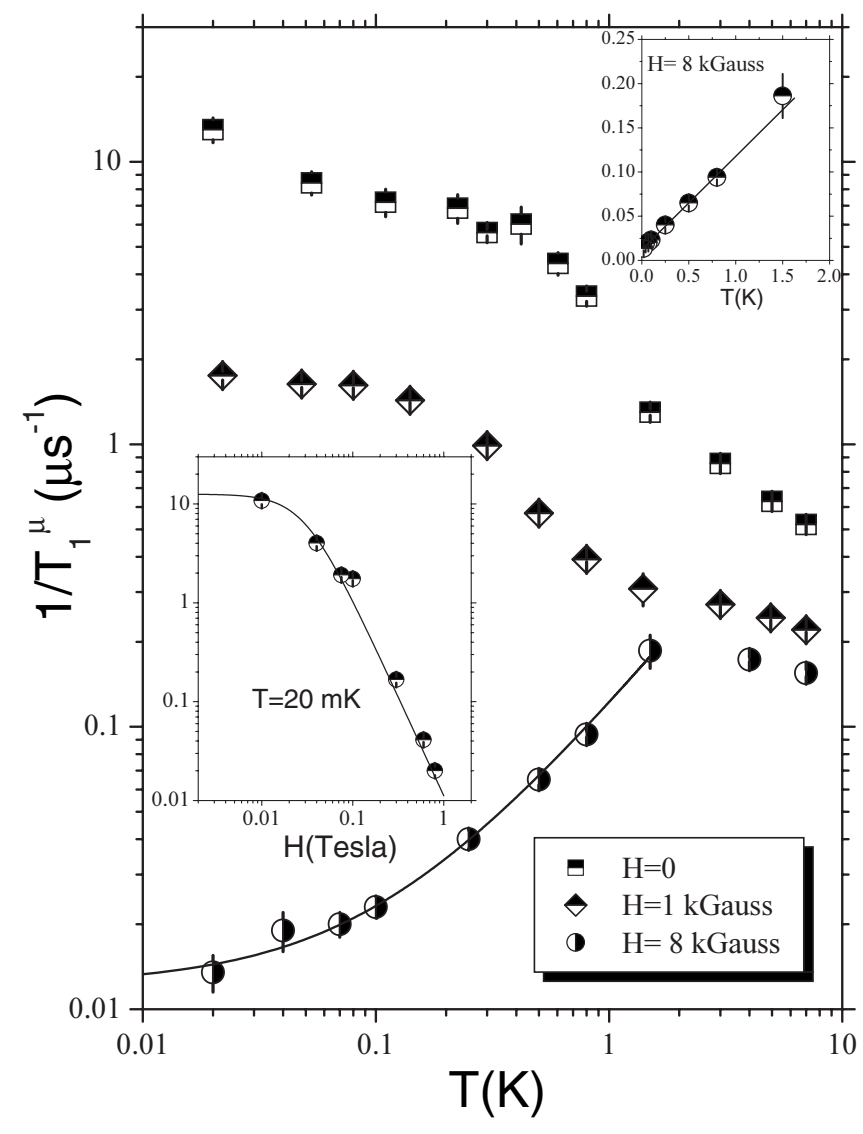

FIG. 4. $T$ dependence of the muon spin-lattice relaxation rate in $\mathrm{YbCu}_{4.4} \mathrm{Au}_{0.6}$ at three different magnetic fields. The solid line and the inset at the top (linear scales) evidence that for $\mathrm{H}=8 \mathrm{kG}$, at low $T, 1 / T_{1}=a T+b$. The small offset $b$ should be associated either with the uncertainty in the background correction or with the fact that a certain angular dependence of the effect of the magnetic field has to be expected. In the lower inset the magnetic field dependence of $1 / T_{1}$ is shown. The solid line shows the scaling law $1 / T_{1}(H)$ $=1 / T_{1}(0)\left[1+\left(\mu_{B} H / k_{B} T\right)^{2}\right]^{-1}$.

Now we turn to the discussion of the effect of a magnetic field on the muon longitudinal relaxation rate $1 / T_{1}^{\mu}$. From Fig. 4 one notices that the magnetic field progressively reduces $1 / T_{1}^{\mu}$. Remarkably the effect is significant at low $T$, where $1 / T_{1}^{\mu}(H=0)$ is large, while it is reduced at high $T$, where $1 / T_{1}^{\mu}(H=0)$ is low. This behavior is the opposite of what one would expect if the relaxation had to be associated with a static field distribution $\Delta H$. In fact, in that case one would expect a significant reduction in the relaxation when $\gamma H \simeq 10 / T_{1}^{\mu}(H=0),{ }^{17}$ at variance with the experimental findings. Moreover, the similar behavior of $1 / T_{1}^{63}$ and $1 / T_{1}^{\mu}$ suggests that the effect of the magnetic field has to be rather associated with a modification in the dynamical spin susceptibility. In fact, in other intermetallic compounds as $\mathrm{CeCu}_{6-x} \mathrm{Au}_{x}$ (Ref. 18) and $\mathrm{YbRh}_{2} \mathrm{Si}_{2}$ (Ref. 19) a similar effect of the magnetic field has been reported. In particular, it has been observed that the magnetic field drives the system away from the QCP toward a FL ground state. Here we observe that at $H=8 \mathrm{kG}, 1 / T_{1}$ decreases linearly with decreasing $T$ (Fig. 4) as expected for a FL. ${ }^{20}$ Our results are perfectly consistent with the experimental findings by Tsujii 
et $a .^{21}$ who observed in $\mathrm{YbCu}_{4.4} \mathrm{Au}_{0.6}$, for $H \simeq 1 \mathrm{~T}$, a crossover in the $T$ dependence of the resistivity from a $T^{3 / 2}$ to a $T^{2}$ power law, the one typical of a FL.

The effect of the magnetic field cannot be explained within SCR theory as an initial raise in $1 / T_{1}$, and then a decrease should be expected. ${ }^{11}$ This is not the case here; in fact, for $H=1 \mathrm{kG} 1 / T_{1}$ increases upon cooling down to the lowest temperature, while for $H=8 \mathrm{kG} 1 / T_{1}$ is practically always decreasing upon cooling. In $\mathrm{CeCu}_{6-x} \mathrm{Au}_{x}$ the effect of the magnetic field was accounted for by a renormalization of the temperature scale to $T_{m}=T\left[1+\left(\mu_{B} H / k_{B} T\right)^{2}\right]^{1 / 2} \cdot{ }^{12,22}$ Here, by taking into account that $1 / T_{1} \propto T \chi_{S}^{2} \propto T^{-1 / 3}$, one should expect that $1 / T_{1}(H) \propto\left[1+\left(\mu_{B} H / k_{B} T\right)^{2}\right]^{-1 / 6}$, at variance with the experimental findings.

On the other hand, we find that the relaxation rate obeys the scaling $1 / T_{1}(H)=1 / T_{1}(0)\left[1+\left(\mu_{B} H / k_{B} T\right)^{2}\right]^{-1}$, with no adjustable parameter (Fig. 4). Accordingly, for $T \rightarrow 01 / T_{1}(H)$ $\propto 1 / H^{2}$. Remarkably also in MnSi (Ref. 23) the muon relaxation rate was observed to decrease with $H^{2}$ upon approaching the transition to the magnetic ground state. Also in that case SCR theory could not explain the field dependence of the relaxation rate and its decrease with $\mathrm{H}^{2}$ was tentatively ascribed to the progressive quenching of the helical components of the critical fluctuations and to the increase in $q=0$ fluctuations. ${ }^{23}$ This explanation, of course, cannot hold here where the critical fluctuations are at $q=0$. The scaling law experimentally found in this work should rather take into account the progressive departure from the ferromagnetic QCP, induced by the magnetic field, and the insurgence of the FL ground state. Finally it has to be pointed out that in spite of the cubic structure the orientation of the magnetic field with respect to $\mathrm{Yb}^{3+}$ crystal-field axes could have some effect.

In conclusion, from the $T$ dependence of the muon and ${ }^{63} \mathrm{Cu}$ nuclear spin-lattice relaxation rates in $\mathrm{YbCu}_{4.4} \mathrm{Au}_{0.6}$ we found that for $T \rightarrow 01 / T_{1}$ diverges, following the behavior predicted by the self-consistent renormalization theory developed by Moriya $^{8}$ for a ferromagnetic quantum critical point and no evidence of any phase transition could be detected down to $20 \mathrm{mK}$. On the other hand, at low $T$ the static uniform susceptibility $\chi_{s}$ was observed to diverge as $T^{-2 / 3}$ and, accordingly, $1 / T_{1} T \propto \chi_{s}^{2}$, a behavior which cannot be explained within SCR theory. Finally, the application of a magnetic field $H$ is observed to induce a crossover to a Fermi-liquid behavior and for $T \rightarrow 01 / T_{1}$ is found to obey the empirical scaling law $1 / T_{1}(H)$ $=1 / T_{1}(0)\left[1+\left(\mu_{B} H / k_{B} T\right)^{2}\right]^{-1}$.

The NMR measurements in Pavia were supported by Fondazione Cariplo 2005 Research Grant.
${ }^{1}$ N. Grewe and F. Steglich, in Handbook on the Physics and Chemistry of Rare Earths, edited by K. A. Geschneider and L. Eyring (North-Holland, Amsterdam, 1991), Vol. 14, p. 343.

${ }^{2}$ Y. Yang, Z. Fisk, H.-O. Lee, J. D. Thompson, and D. Pines, Nature (London) 454, 611 (2008).

${ }^{3}$ See, for instance, O. Stockert, F. Huster, A. Neubert, C. Pfeiderer, T. Pietrus, B. Will, and H. v. Löhneysen, Physica B 312-313, 458 (2002); K. Heuser, E.-W. Scheidt, T. Schreiner, and G. R. Stewart, Phys. Rev. B 57, R4198 (1998).

${ }^{4}$ K. Yoshimura, T. Kawabata, N. Sato, N. Tsujii, T. Terashima, C. Terakura, G. Kido, and K. Kosuge, J. Alloys Compd. 317-318, 465 (2001).

${ }^{5}$ M. Galli, E. Bauer, St. Berger, Ch. Dusek, M. Della Mea, H. Michor, D. Kaczorowski, E. W. Scheidt, and F. Marabelli, Physica B 312-313, 489 (2002).

${ }^{6}$ M. Giovannini, A. Saccone, St. Müller, H. Michor, and E. Bauer, J. Phys.: Condens. Matter 17, S877 (2005)

${ }^{7}$ M. Giovannini, R. Pasero, S. De Negri, and A. Saccone, Intermetallics 16, 399 (2008).

${ }^{8}$ T. Moriya, Spin Fluctuations in Itinerant Electron Magnetism (Springer, Berlin, 1985).

${ }^{9}$ R. Abela, A. Amato, C. Baines, X. Donath, R. Erne, D. C. George, D. Herlach, G. Irminger, I. D. Reid, D. Renker, G. Solt, D. Suhi, M. Werner, and U. Zimmermann, Hyperfine Interact. 120-121, 575 (1999).

${ }^{10}$ N. Tsujii, K. Yoshimura, and K. Kosuge, Phys. Rev. B 59, 11813 (1999).

${ }^{11}$ A. Ishigaki and T. Moriya, J. Phys. Soc. Jpn. 65, 3402 (1996).
${ }^{12}$ A. Schröder, G. Aeppli, R. Coldea, M. Adams, O. Stockert, H. v. Löhneysen, E. Bucher, R. Ramazshavili, and P. Coleman, Nature (London) 407, 351 (2000).

${ }^{13}$ P. Gegenwart, J. Custers, Y. Tokiwa, C. Geibel, and F. Steglich, Phys. Rev. Lett. 94, 076402 (2005).

${ }^{14}$ E. Bauer, P. Fischer, F. Marabelli, M. Ellerby, K. A. McEwen, B. Roessli, and M. T. Fernandes-Dias, Physica B 234-236, 676 (1997).

${ }^{15}$ A. Ishigaki and T. Moriya, J. Phys. Soc. Jpn. 67, 3924 (1998).

${ }^{16}$ M. Corti, F. Carbone, M. Filibian, Th. Jarlborg, A. A. Nugroho, and P. Carretta, Phys. Rev. B 75, 115111 (2007).

${ }^{17}$ S. J. Blundell, Contemp. Phys. 40, 175 (1999).

${ }^{18}$ P. Carretta, M. Giovannini, M. Horvatic, N. Papinutto, and A. Rigamonti, Phys. Rev. B 68, 220404(R) (2003).

${ }^{19}$ K. Ishida, K. Okamoto, Y. Kawasaki, Y. Kitaoka, O. Trovarelli, C. Geibel, and F. Steglich, Phys. Rev. Lett. 89, 107202 (2002).

${ }^{20}$ C. P. Slichter, Principles of Magnetic Resonance, 3rd ed. (Springer, Berlin, 1990).

${ }^{21}$ N. Tsujii, T. Terashima, C. Terakura, G. Kido, T. Kawabata, K. Yoshimura, and K. Kosuge, J. Phys.: Condens. Matter 13, 3623 (2001).

${ }^{22}$ P. Carretta, M. Giovannini, M. J. Graf, N. Papinutto, A. Rigamonti, and K. Sullivan, Physica B 378-380, 84 (2006).

${ }^{23}$ I. M. Gat-Malureanu, A. Fukaya, M. I. Larkin, A. J. Millis, P. L. Russo, A. T. Savici, Y. J. Uemura, P. P. Kyriakou, G. M. Luke, C. R. Wiebe, Y. V. Sushko, R. H. Heffner, D. E. Mac Laughlin, D. Andreica, and G. M. Kalvius, Phys. Rev. Lett. 90, 157201 (2003). 